\title{
A CONTRACORRIENTE. TRABAJADORES Y EMPRESARIOS CHINOS EN ESPAÑA ANTE LA CRISIS ECONÓMICA (2007-2013) ${ }^{1}$
}

\section{AGAINST THE TIDE. CHINESE WORKERS AND ENTREPRENEURS IN SPAIN FACING THE ECONOMIC CRISIS (2007-2013)}

Joaquín Beltrán Antolín y Amelia SÁlz López*

Resumen: A partir de una comparación de los efectos de la crisis económica (2007-2013) en España sobre la población y trabajadores nacionales y extranjeros con los de origen chino, se pone de manifiesto la conducta diferencial de estos últimos, a contracorriente de la tendencia de pérdida de empleo. Las y los trabajadores y empresarios chinos aumentan de forma constante consolidando y profundizando estrategias previas de su inserción en el mercado laboral español mediante el desarrollo de nichos económicos étnicos donde la propiedad de empresas de carácter familiar es el modelo dominante. La coincidencia del comienzo de la crisis económica (2007) con la apertura de un nuevo nicho étnico a la espera de su expansión, con una reducción de los costes iniciales de sus negocios, ha permitido una aceleración en el acceso a la propiedad de empresas familiares.

* Universidad Autónoma de Barcelona.

1 Este artículo se enmarca en el proyecto de investigación I + D MINE$\mathrm{CO}$ «El impacto de Asia Oriental en el contexto español: Producción cultural, política(s) y sociedad» (FFI2011-29090) del grupo de Investigación InterAsia (2014SGR1402, AGAUR) de la Universidad Autónoma de Barcelona. Así como en el proyecto "Género, transnacionalismo y estrategias intergeneracionales de movilidad social» (FEM2011-26210) 
Palabras clave: crisis económica y migración; migración china; empresariado chino; nichos económicos; chinos en España

Abstract: From a comparison of the effects of the economic crisis (2007-2013) in Spain on the national population and foreign workers and those of Chinese origin, highlights the differential behavior of the latter, against the tide of job loss. The Chinese workers and entrepreneurs increase steadily consolidating and deepening strategies prior to insertion into the Spanish labor market through the development of ethnic economic niches where ownership of family business is the dominant model. The coincidence of the beginning of the economic crisis (2007) with the opening of a new ethnic niche waiting to expand, with a reduction of the initial costs of their business, has allowed an acceleration in access to ownership of family business.

Keywords: migratory strategies; migratory project; return; migratory perspective; social reproduction.

Los residentes y trabajadores chinos en España se diferencian del resto de los inmigrantes extranjeros en su relación con la actividad económica durante la reciente crisis (2007-2013). En este artículo se analizará en primer lugar cómo ha afectado la crisis económica a la inmigración en España en términos de empleo, para a continuación presentar las características de las actividades económicas de los inmigrantes chinos antes y durante de la crisis, con especial atención a las trayectorias empresariales y estrategias desarrolladas para afrontarla.

La metodología utilizada incluye además de una revisión de estudios publicados sobre inmigración y crisis económica en España, el análisis de fuentes estadísticas oficiales sobre residencia y trabajo de los extranjeros, así como datos del trabajo de campo realizado por los autores durante más de veinte años de investigación sobre la inmigración china en España, incluyendo estudios específicos sobre empresariado asiático.

\section{INMIGRACIÓN Y CRISIS ECONÓMICA EN ESPAÑA}

La crisis económica iniciada a finales del año 2007 en España ha tenido, entre otras consecuencias un aumento generalizado 
del desempleo y una disminución de la actividad económica y del crecimiento de la riqueza del país. La mano de obra extranjera ha descendido considerablemente después de un extraordinario crecimiento durante los años precedentes. Esta situación ha provocado finalmente que el balance migratorio internacional haya pasado a ser negativo.

Durante 30 años (1965-1995) la población ocupada en España se mantuvo en torno a 12 millones de personas. ${ }^{2}$ En 1997 había 12,9 millones de ocupados y desde entonces hasta el año 2007 la ocupación aumentó en 7,5 millones, hasta alcanzar 20,4 millones de empleos ese año. Paralelamente, la tasa de desempleo que llegó a su cénit en 1994 con un 24,5\% del total de la población activa $(3,9$ millones de personas), fue disminuyendo progresivamente hasta su punto más bajo en 2007 del 7,9\%. El ciclo expansivo económico caracterizado por un rápido aumento de la población ocupada junto a la reducción de la tasa de desempleo hasta un mínimo sin precedentes se detuvo con el inicio de la crisis, para comenzar a disminuir de modo también acelerado: en el primer trimestre de 2014 los ocupados eran 16,9 millones y los desempleados 5,9 millones (25,9\% del total). Por otra parte, no se puede explicar el ciclo expansivo sin la llegada de inmigrantes procedentes del extranjero especialmente a partir de 1998. Y así, por ejemplo del año 2003 al 2008 inclusive el balance migratorio en España superaba anualmente el medio millón de extranjeros, convirtiendo al país durante un lustro en el segundo destino más importante para los movimientos de migración internacional después de EEUU.

En el contexto de la crisis económica el balance migratorio se reduce y ha pasado de un saldo positivo de 310.642 en 2008 a otro de 12.845 en 2009 , para convertirse en negativo por primera vez en 2010 (-42.675) y llegar a -142.552 en 2012. Es en 2010, cuando definitivamente emigran más extranjeros de los que entran. La crisis económica que se desencadena a finales de 2007 en España, y en otros países europeos, ha afectado a los residentes, trabajadores y empresarios extranjeros de diferente modo. Sin duda, el ritmo de crecimiento y llegada de población y de trabajadores ha disminuido,

2 Los datos estadísticos de población, trabajo y migración que aparecen en este apartado proceden de distintas secciones del Instituto Nacional de Estadística, especialmente de «Mercado Laboral»: Encuesta de Población Activa (EPA); y de «Demografía y población»: Padrón, Censos y Migraciones. 
pero su pérdida significativa no se produce hasta el año 2012, casi cinco años después de comenzar la crisis.

¿Por qué? La segmentación del mercado laboral español, el mercado dual y la complementariedad de la fuerza de trabajo autóctona y extranjera (Gil-Alonso y Domingo, 2008), se pone de manifiesto cuando la mayoría de los inmigrantes desempeñan los trabajos de menor cualificación, más temporales y peor pagados, es decir, los puestos de trabajo que procura evitar la población autóctona y que se caracterizan por una mayor precariedad. Durante la crisis este tipo de puestos de trabajo han sido los últimos en desaparecer - salvo alguna excepción como en el sector de la construcción que fue uno de los primeros afectados por la misma (Vidal-Coso, Gil-Alonso y Domingo, 2012; Oliver, 2013). De hecho, con la crisis no sólo ha aumentado la tasa de desempleo (Medina, Herrarte y Vicéns, 2010; Esteban, 2011), sino que también se ha perdido una parte significativa de los «mejores» trabajos aumentando la precariedad laboral - de contratos temporales, entre otras cosas-, el crecimiento de la tasa de actividad femenina (de 48,6\% en 2007 a 53,3\% en 2013) y la reducción de la masculina (68,9\% a $65,9 \%$ en las mismas fechas). Los inmigrantes ya ocupaban los peores puestos de trabajo antes de la crisis, y ha sido a estos últimos a los que más tarde les han llegado sus consecuencias (Colectivo Ioé, 2012; Valero-Matas, Coca y Valero-Oteo, 2014).

Zugasti Mutilva (2014) en su análisis sobre la población extranjera que accede a un contrato indefinido durante el periodo 20062010 , señala que

«Las personas no nacionales tienen dificultades para acceder al empleo indefinido en el contexto español y, cuando lo consiguen, muestran un mayor riesgo ante la pérdida de dicho empleo. Sin embargo, en un momento en el que el mercado de trabajo se encuentra en estado de conmoción, también se producen resistencias en la integración en el empleo de calidad e incluso aumenta el número de personas extranjeras que consigue mantener su contrato indefinido» (Zugasti, 2014: 302).

En cinco años, de 2008 a 2013, la Seguridad Social ha perdido 486.522 altas de trabajadores extranjeros (22,8\% de disminución) incluidas en la pérdida global de 2.965 .088 altas de trabajadores en 
España (15,3\% de disminución). En ese mismo periodo se ha producido un aumento de 3,6 millones de desempleados registrados.

En este contexto económico, el comportamiento laboral a contracorriente de los y las trabajadores de origen chino en España ha mantenido un crecimiento continuado de su volumen y de sus actividades empresariales, un fenómeno característico de su inserción laboral en el sur de Europa, especialmente de los procedentes del distrito de Qingtian (provincia de Zhejiang) que constituyen la mayor parte de su población en los países de la zona (España, Italia, Portugal) y dominan de forma hegemónica el modelo y las estrategias que siguen el resto.

\section{POBLACIÓN CHINA Y TRABAJO CON ANTERIORIDAD A LA CRISIS ECONÓMICA}

La presencia china en España cuenta con una relativamente larga historia, aunque su volumen, al igual que el de otros colectivos extranjeros, no es realmente significativo hasta la entrada de España en la Comunidad Económica Europea en 1986. El ingreso en este organismo supuso un rápido aumento del nivel de vida de la sociedad española, y oportunidades de trabajo para extranjeros en determinados sectores económicos que no competían con el mercado laboral primario o general.

A finales de la década de 1980, España era un territorio virgen para establecer restaurantes de comida china cuando, por fin, la población española comenzó a frecuentarlos. Tras el establecimiento de relaciones diplomáticas con la República Popular China en 1973, Chen Diguang, de Fushan (Qingtian), se reunificó en 1974 con su tío Chen Tse-ping —asentado en España desde la década de 1940 y dueño del Teatro Chino de Manolita Chen-, comenzó a trabajar en un restaurante de comida china taiwanés en Madrid y en 1975 abrió su primer restaurante, el Gran Muralla, pasando con el tiempo a detentar una pequeña cadena de 15 restaurantes de comida china y ayudando a más de 300 parientes a emigrar a España, todos ellos acabaron trabajando en el negocio de la restauración de comida china. El control taiwanés de la escasa oferta de este tipo de restaurantes en las grandes capitales (Madrid y Barcelona) hasta mediados de la década de 1980 pronto tuvo que hacer frente a la llegada y 
establecimiento, ahora ya directamente desde China, de nuevos inmigrantes procedentes de Qingtian que abrieron más restaurantes.

Históricamente, el restaurante de comida china, en algunos países del norte de Europa y posteriormente del sur también, ha sido un modelo de empresa familiar para la supervivencia y prosperidad de sus miembros. Esta actividad económica se ha explotado hasta llegar al punto de saturación de su oferta. En España ocurre a mediados de la década de 1990, momento en que la primera dispersión china por todo el territorio llega a los municipios con una población o ámbito de influencia de 8.000 habitantes, gracias al establecimiento de este tipo de restaurantes. La población china pasó de 2.455 personas en 1986 a 10.816 en 1996. Una parte importante eran menores de 15 años, bien nacidos en España o llegados mediante procesos de reunificación familiar; ambas tendencias continúan hasta la actualidad. En el año 2000 las y los residentes chinos en España ya sumaban 28.693 y, al igual que en el resto de los colectivos extranjeros, su volumen creció de forma muy rápida hasta el comienzo de la crisis: 119.859 residentes chinos en 2007 $(19,9 \% \text { menores de } 15 \text { años) })^{3}$.

El modelo de empresa familiar chino en España se basa en asegurar la propiedad familiar de los medios de producción - por ejemplo, el restaurante de comida china-, donde la mano de obra fundamental son los propios miembros de la familia - padres e hijos, otros parientes-. El restaurante es, al mismo tiempo, un centro de formación para los parientes inmigrantes recién llegados que comienzan a trabajar en él y con la práctica y experiencia aprenden todo el proceso de funcionamiento del negocio hasta que logran independizarse y abrir un restaurante propio donde vuelve a repetirse el proceso. De este modo se desarrolla un nicho étnico, es decir, un sector de actividad económica concreta donde la propiedad de los negocios y/o la fuerza de trabajo es fundamentalmente coétnica (Light, 2006; Veira, Stanek y Cachón, 2011). Dentro del nicho étnico, además de la formación en el mismo sector, también se obtienen créditos para completar el capital inicial cuando el ahorro acumulado mediante el trabajo asalariado no es suficiente (Beltrán, 2000).

3 Estos datos proceden de las estadísticas de permisos de residencia de extranjeros del Ministerio de Interior. 
Una vez que una oferta se satura y ante la presión de la llegada desde China de más mano de obra disponible, los empresarios con más años de residencia y, por tanto más experiencia y conocimiento sobre el país, se aventuran invirtiendo en nuevos sectores de actividad y acaban desarrollando nuevos nichos étnicos donde la lógica económica original se repite, en la medida en que demuestra su eficacia. Durante los años noventa, las y los trabajadores chinos también aparecieron en el sector secundario mediante los talleres textiles para la confección de ropa. Este tipo de trabajo, situado en la escala más baja de la jerarquía laboral china en España, se ha caracterizado por ser temporal, con una extraordinaria movilidad de la mano de obra. Una oferta empresarial que, para mediados de la primera década del siglo XXI muestra señales de agotamiento a diferencia de su experiencia en Italia en este sector (Ceccagno, 2007). Su inserción en la construcción, bien sea trabajando para empleadores chinos o españoles, ha sido en un volumen y un tiempo relativamente corto.

Por lo que respecta al trabajo chino asalariado para empleadores autóctonos hay que mencionar su inserción en todo tipo de industrias - las industrias cárnicas en Olot en Gerona, por ejemplo-, agricultura, e incluso servicios — camareros de bares-. Sin embargo, en conjunto su presencia como asalariados, o en los sectores anteriormente referidos, ha sido minoritaria en comparación con su establecimiento y empleo en el sector de la restauración, el comercio y los servicios de proximidad. Entre ellos, sobresalen los bazares, también conocidos como tiendas de «todo a cien [pesetas]» o tiendas de "todo a un euro», que surgen a mediados de los noventa, llegando a su consolidación y saturación durante el primer lustro del siglo xxI, componiendo la segunda ola de dispersión china por toda la geografía española. Estos bazares van asociados a empresas grandes y medianas de importación/exportación que cuentan con grandes almacenes de venta al por mayor habitualmente localizados en polígonos industriales, pero a veces también en zonas céntricas de las ciudades - Lavapiés en Madrid, Arco de Triunfo en Barcelona, barrio de la estación de autobuses en Alicante, etc.- . Algunos de estos importadores se han especializado en ropa confeccionada, o en zapatos y marroquinería, dando lugar también a la apertura de pequeñas tiendas de ropa y de zapaterías.

Ante la falta de censos sistemáticos de negocios por origen étnico en España, valga como indicador de su actividad empresarial el siguiente testimonio del año 2005: 
«[De acuerdo a Song Hua Xu, presidente de la Asociación de Chinos en España]: Hay 4.000 restaurantes chinos en España, 3.200 tiendas de todo a cien, 1.500 establecimientos de frutos secos, 600 almacenes mayoristas, 80 tiendas donde se vende exclusivamente comida china, 200 talleres textiles y 120 establecimientos de revelado de fotos. También hay un número menor de establecimientos como peluquerías o tiendas de ordenadores. En total habrá más de 10.000 empresas. No tenemos paro. Buena parte de las empresas están formadas por dos personas. Tenemos muchos empleados españoles» (Luis Gómez, «El poder chino en España», El País, 27 de marzo de 2005).

En este párrafo encontramos descritas las características principales de las ocupaciones del colectivo chino en España en ese momento. En su mayor parte se trata de pequeñas empresas familiares cuya oferta en algún caso va también destinada a los coétnicos, por ejemplo, los supermercados de comida china. Conforme el volumen de la población china aumenta, también lo hacen los negocios abiertos para satisfacer sus necesidades como agencias de viaje, agencias inmobiliarias, escuelas para aprender a conducir, academias privadas para estudiar español y enseñar chino a las y los niños, estudios de fotografía, tiendas de alquiler de trajes de novia, joyerías, floristerías, tiendas de medicina china, clínicas de medicina china y de biomedicina con médicos chinos, pensiones y hoteles, restaurantes de comida china rápida y regional, tiendas de música, cine, revistas y libros en chino, explotaciones agrarias para cultivar vegetales chinos, crianza de patos, fábricas de doufu, etc. Las oportunidades para nuevos negocios se multiplican, no obstante, en la medida en que la clientela coétnica es siempre limitada, continúa la exploración de nuevos sectores y nichos económicos donde abrir empresas familiares destinadas a una clientela general.

La llegada continuada de nueva mano de obra procedente de China, bien sea por medio de ofertas de contrato de trabajo, por reunificaciones familiares o por entradas irregulares, no cesa. Igual que tampoco se detienen sus expectativas empresariales, siendo uno de los colectivos extranjeros que cuenta con una proporción más elevada de trabajadores autónomos entre sus afiliados a la Seguridad Social. La lógica económica del nicho étnico, facilita la formación en el sector mediante la práctica laboral, el acceso a la información clave para decidir el momento y lugar de apertura, y la ayuda para la financiación — fundamentalmente de familiares y amigos- del 
establecimiento de los negocios, e incluso se asume el fracaso de las iniciativas empresariales fallidas, puesto que no todos los negocios tienen éxito, posibilitando un nuevo ciclo empresarial en el seno del nicho étnico.

La familia-empresa sigue al ciclo de vida familiar, e idealmente, los negocios pasan por las fases de apertura, consolidación y expansión conforme la familia aumenta sus miembros bien por matrimonio, por nacimiento o por traspaso de una generación a la siguiente. Dentro de esta lógica económica del nicho étnico también es sobresaliente la presencia de mujeres empresarias. De hecho, el porcentaje de trabajadoras autónomas chinas suele superar al de los hombres chinos (Sáiz López, 2012, 2013).

\section{GRÁFICO 1}

TRABAJADORES CHINOS POR SECTORES DE ACTIVIDAD. AÑOS 1991-2008 (EN \%)

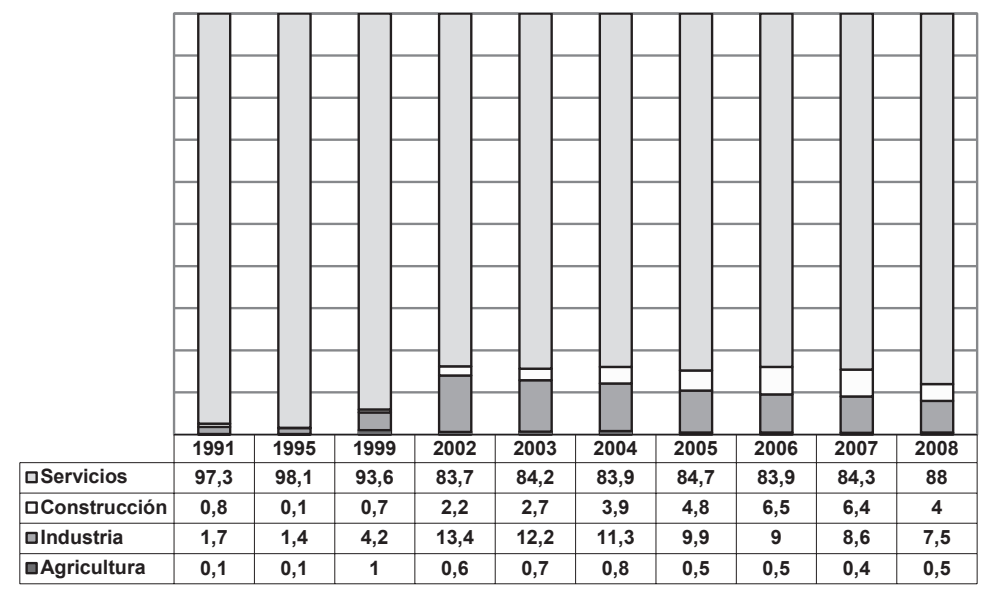

Fuente: Elaboración propia a partir del Anuario de Migraciones y Anuario de Estadísticas Laborales y de Asuntos Sociales.

En definitiva, para el año 2007 en España existe una población china con una gran vitalidad empresarial, sobre todo en el ámbito de los servicios donde se ha insertado en varios sectores que explota hasta llegar al punto de saturación de su oferta. El modelo 
económico se caracteriza por la gran movilidad de las empresas familiares, la dispersión geográfica y la diversificación de las mismas, es decir, por su gran flexibilidad.

Uno de los itinerarios más característicos de estos años se encuentra en los empresarios de restaurantes de comida china. Cuando han logrado el éxito económico, o bien lo venden o lo traspasan para invertir en empresas de importación/exportación, el negocio que se convierte en el nuevo símbolo del prestigio social y económico dado que son las que más capital movilizan y beneficios obtienen. Algunas están formadas por varios socios que aportan capital y/o mano de obra. Otra modalidad de diversificación implica la incursión en otro sector sin abandonar el primero donde se invirtió.

La aparición de China en el panorama económico mundial ha favorecido y ampliado la bidireccionalidad de las inversiones en el espacio económico transnacional chino. A las remesas y capital destinado a la creación y mejora de las infraestructuras locales, hay que añadir la inversión en nuevas actividades productivas y especulativas, en el lugar de origen y en el resto del país, sin abandonar la base empresarial ya establecida en España, un capítulo financiero de las empresas familiares chinas anteriormente de menor relevancia.

En suma, con el paso del tiempo, las familias chinas han incorporado y consolidado prácticas y saberes que se añaden a los ya existentes en la cultura migratoria local, se da un proceso de diversificación a escala local, nacional y global, y se mantiene la movilidad y flexibilidad empresarial en función de la rentabilidad, buscando nuevas oportunidades, y clausurando las empresas que han dejado de proporcionar beneficios.

\section{ACTIVIDADES ECONÓMICAS Y LABORALES EN LOS AÑOS DE LA CRISIS}

Desde el año 2007 hasta el 2013 la población china, no ha dejado de aumentar y de rejuvenecer: los 119.859 residentes chinos de finales de 2007 pasan a 184.196 en 2013, es decir en seis años aumentan en 64.337 personas, de las cuales 23.493 son menores de 15 años, segmento de edad que aumenta 5,8 puntos porcentuales durante 
este periodo (de $19,9 \%$ a $25,7 \%)^{4}$. También es significativo el incremento de la proporción de los residentes chinos nacidos en España; según los datos de padrón continuo del INE, del 10,3\% del total a comienzos de 2007 se pasa al 18\% a comienzos de 2014. De este modo, el perfil actual es el de una población joven —el promedio de edad es de 29 años-, bastante equilibrada por sexo — $48 \%$ mujeres frente a $52 \%$ hombres-, compuesta fundamentalmente por matrimonios jóvenes con hijos menores de edad, dueños de un negocio. El crecimiento anual de su población ha disminuido en números absolutos en comparación con años anteriores, pero no se ha interrumpido: de 2010 a 2011, aumenta en 13.800 personas; y de finales de 2012 a finales de 2013 el aumento ha sido de 5.078 personas. Es uno de los pocos colectivos extranjeros que ha incrementado su población residente en España en los últimos años.

GRÁFICO 2

\section{ESTRUCTURA DE EDAD DE LA POBLACIÓN RESIDENTE CHINA EN ESPAÑA. AÑOS 2007-2013}

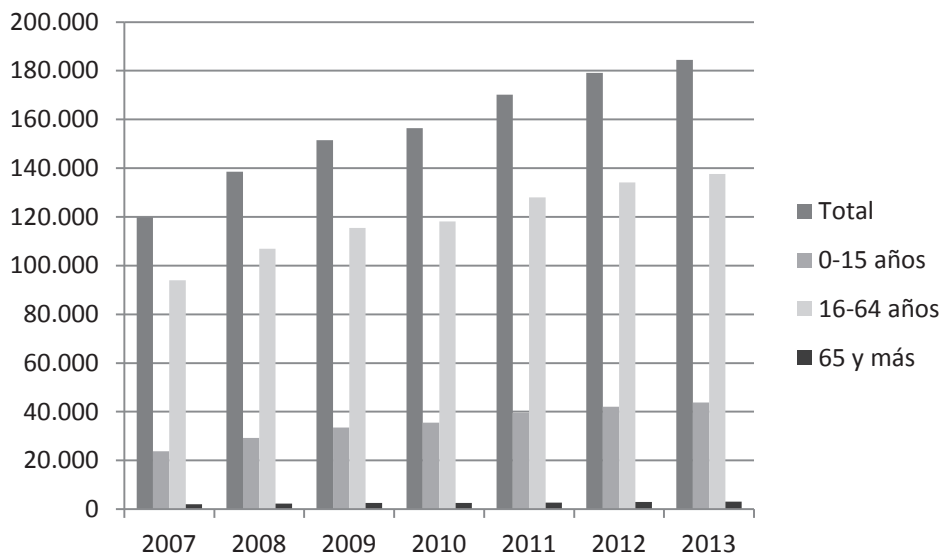

Fuente: Elaboración propia a partir de los Boletines de Inmigración. MTSS.

4 Datos procedentes de los boletines de «Extranjeros residentes en España» del Ministerio de Empleo y Seguridad Social. 
El incremento de la población de origen chino y la saturación de la oferta de sus principales nichos étnicos - restaurantes de comida china, bazares - ya en el año 2007, ha supuesto su inserción en nuevos sectores de actividad económica, especialmente en servicios donde han aumentado y diversificado su presencia. Los nuevos nichos, dejando aparte el desarrollo y rápida evolución de las peluquerías, están relacionados con la restauración y la hostelería, sectores en los que se observa:

1. La diversificación de la comida "étnica» con la aparición de restaurantes de comida japonesa, de comida «asiática»; la reinvención del restaurante chino tradicional en el formato wok o comida a la vista de grandes dimensiones; aumento de la oferta especializada de comida regional china, así como de comida rápida china, etc.

2. El traspaso y apertura de bares y restaurantes españoles sin cambios en la oferta culinaria. Este segundo desarrollo es el más importante, pues ha abierto un sector a la iniciativa empresarial china notable dada la peculiaridad de los hábitos de consumo españoles que tienen al bar y cafetería como centros de articulación social de primer orden, hasta el punto de que España es el país con más bares, cafeterías y restaurantes per cápita del mundo - 1 bar por cada 150 personas aproximadamente— sólo superado por Chipre.

La mayoría de los bares en España son micro-bares, pequeñas empresas familiares que no tienen recambio generacional. Es decir, cuando llega el momento de la jubilación de los propietarios, la siguiente generación - los hijos e hijas-, no desean seguir con la empresa familiar. La alternativa es el cierre de los mismos quedando su clientela habitual sin un servicio al que estaba acostumbrada. Ante esta situación los trabajadores chinos traspasan los bares y los continúan gestionando como lo que eran, pequeñas empresas familiares, dando continuidad a un negocio con una clientela a la que se le continúa ofreciendo el mismo servicio. Así, el éxito radica en no cambiar la oferta de servicio (Beltrán y Sáiz, 2013). 


\section{TABLA 1}

\section{TRABAJADORES EXTRANJEROS DADOS DE ALTA EN LA SEGURIDAD SOCIAL, AÑOS 2006-2013}

\begin{tabular}{|l|c|c|c|c|c|}
\cline { 2 - 6 } \multicolumn{1}{c|}{} & $\begin{array}{c}\text { Total } \\
\text { trabajadores } \\
\text { extranjeros }\end{array}$ & $\begin{array}{c}\text { \% variación } \\
\text { interanual }\end{array}$ & $\begin{array}{c}\text { Total } \\
\text { trabajadores } \\
\text { autónomos }\end{array}$ & $\begin{array}{c}\text { \% variación } \\
\text { interanual }\end{array}$ & $\begin{array}{c}\% \text { autónomos } \\
\text { sobre el total }\end{array}$ \\
\hline $31 / 12 / 2006$ & 1.823 .974 & & 164.630 & & $9,0 \%$ \\
\hline $31 / 12 / 2007$ & 1.981 .106 & $8,6 \%$ & 223.597 & $35,8 \%$ & $11,3 \%$ \\
\hline $31 / 12 / 2008^{\star}$ & 1.938 .632 & $-2,1 \%$ & 224.156 & $0,2 \%$ & $11,6 \%$ \\
\hline $31 / 12 / 2009^{\star}$ & 1.848 .047 & $-4,7 \%$ & 197.625 & $-11,8 \%$ & $10,7 \%$ \\
\hline $31 / 12 / 2010^{\star}$ & 1.814 .979 & $-1,8 \%$ & 198.932 & $0,7 \%$ & $11,0 \%$ \\
\hline $31 / 12 / 2011^{*}$ & 1.738 .922 & $-4,2 \%$ & 208.512 & $4,8 \%$ & $12,0 \%$ \\
\hline $31 / 12 / 2012^{\star}$ & 1.645 .851 & $-5,3 \%$ & 215.065 & $3,1 \%$ & $13,1 \%$ \\
\hline $31 / 12 / 2013^{*}$ & 1.543 .306 & $-6,2 \%$ & 223.978 & $4,1 \%$ & $14,5 \%$ \\
\hline
\end{tabular}

* Promedio del mes

Fuente: Elaboración propia a partir de boletines mensuales de Afiliación de Extranjeros a la Seguridad Social. Secretaria de Estado de la Seguridad Social.

Durante la crisis (2007-2013), a pesar de que el número de trabajadores extranjeros dados de alta en la Seguridad Social disminuye de forma progresiva y continuada desde un máximo de 1.981 .106 a finales de 2007, hasta el mínimo de 1.543 .306 a finales de 2013, la proporción de trabajadores autónomos extranjeros crece desde el $9 \%$ del total a finales de 2006 al 14,5\% en 2013. Se ha argumentado que en situaciones de crisis aumenta el autoempleo como estrategia refugio ante la falta de alternativas de acceso al empleo asalariado en el mercado laboral general (Oso y Villares, 2005; Solé y Parella, 2009), fenómeno que se constata a partir de los datos estadísticos (véanse Tabla 1 y Gráfico 3), pero sólo para el caso de los trabajadores extranjeros, pues para el total nacional la variación porcentual apenas es significativa: a finales de 2006 había 3.437.223 trabajadores por cuenta propia en España $(18,2 \%$ del total) que pasan a ser 3.063 .787 a finales de 2013 (18,7\% del total). De cualquier modo, para el colectivo chino, lejos de ser una estrategia refugio, constituye su modo paradigmático de inserción laboral y económica, acorde con su cultura migratoria de movilidad social ascendente (Beltrán, 2000; Beltrán y Sáiz 2009; Sáiz López, 2012). 
GRÁfICO 3

TOTAL TRABAJADORES, EXTRANJEROS

Y CHINOS AUTÓNOMOS. AÑOS 2006-2013

(EN PORCENTAJE SOBRE EL TOTAL)

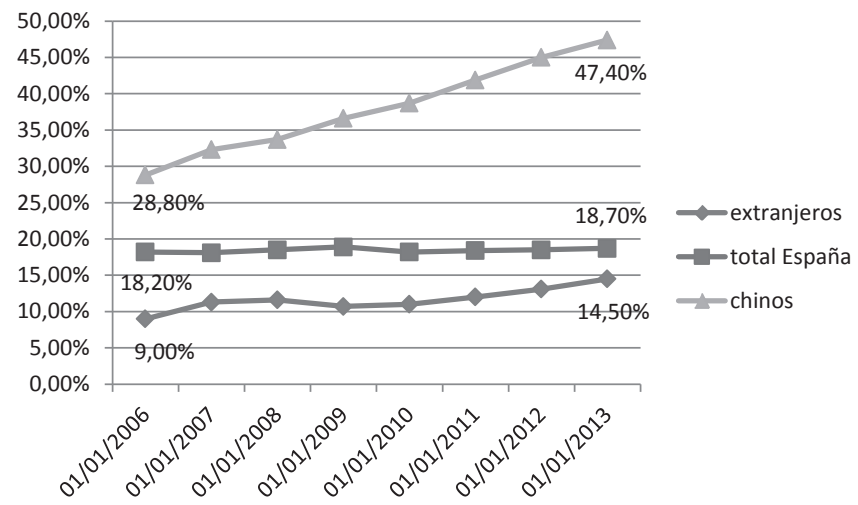

Fuente: Elaboración propia a partir de boletines mensuales de Afiliación de Extranjeros a la Seguridad Social. Secretaria de Estado de la Seguridad Social.

TABLA 2

TRABAJADORES CHINOS DADOS DE ALTA EN LA SEGURIDAD SOCIAL, AÑOS 2006-2013

\begin{tabular}{|l|c|c|c|c|c|}
\cline { 2 - 6 } \multicolumn{1}{c|}{} & $\begin{array}{c}\text { Total } \\
\text { trabajadores } \\
\text { chinos }\end{array}$ & $\begin{array}{c}\text { \% variación } \\
\text { interanual }\end{array}$ & $\begin{array}{c}\text { Total } \\
\text { trabajadores } \\
\text { chinos } \\
\text { autónomos }\end{array}$ & $\begin{array}{c}\text { \% variación } \\
\text { interanual }\end{array}$ & $\begin{array}{c}\% \\
\text { autónomos } \\
\text { sobre el } \\
\text { total }\end{array}$ \\
\hline $31 / 12 / 2006$ & 59.063 & & 16.998 & & $28,8 \%$ \\
\hline $31 / 12 / 2007$ & 63.399 & $7,3 \%$ & 20.501 & $20,6 \%$ & $32,3 \%$ \\
\hline $31 / 12 / 2008^{\star}$ & 71.129 & $12,2 \%$ & 24.005 & $17,1 \%$ & $33,7 \%$ \\
\hline $31 / 12 / 2009^{\star}$ & 75.238 & $5,8 \%$ & 27.508 & $14,6 \%$ & $36,6 \%$ \\
\hline $31 / 12 / 2010^{\star}$ & 83.331 & $10,7 \%$ & 32.285 & $17,4 \%$ & $38,7 \%$ \\
\hline $31 / 12 / 2011^{\star}$ & 87.196 & $4,6 \%$ & 36.541 & $13,2 \%$ & $41,9 \%$ \\
\hline $31 / 12 / 2012^{\star}$ & 88.203 & $1,1 \%$ & 39.718 & $8,7 \%$ & $45,0 \%$ \\
\hline $31 / 12 / 2013^{\star}$ & 89.786 & $1,8 \%$ & 42.559 & $7,1 \%$ & $47,4 \%$ \\
\hline
\end{tabular}

* Promedio del mes

Fuente: Elaboración propia a partir de boletines mensuales de Afiliación de Extranjeros a la Seguridad Social. Secretaria de Estado de la Seguridad Social. 
El colectivo chino cuenta con un elevado porcentaje de pequeños empresarios - 48,7\% son trabajadores autónomos en marzo de 2014-, algo poco habitual entre los trabajadores extranjeros, a excepción de los daneses — 41,6\% autónomos- y algunos otros grupos europeos - holandeses, alemanes, austriacos, belgas, británicos y suecos que cuentan con más del 30\% de trabajadores autónomos-, y asiáticos — coreanos, por ejemplo—.

GRÁFICO 4

TOTAL TRABAJADORES AUTÓNOMOS CHINOS EN ESPAÑA. AÑOS 2007-2013

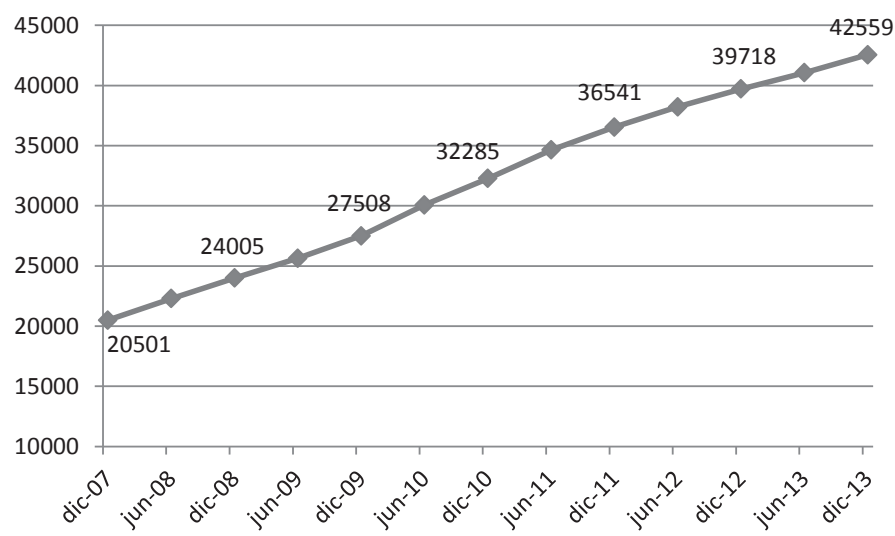

Fuente: Elaboración propia a partir de boletines mensuales de Afiliación de Extranjeros a la Seguridad Social. Secretaria de Estado de la Seguridad Social.

La distribución geográfica de su asentamiento a partir de las estadísticas de trabajadores dados de alta en la Seguridad Social muestra cómo el mayor porcentaje de autónomos se encuentra en las Comunidades Autónomas donde más tarde se han asentado y esto es el resultado de la lógica del nicho económico que pasa de empresarios veteranos en un sector y en ciudades concretas que después se dispersan por todo el territorio nacional. 
TABLA 3

TRABAJADORES CHINOS DADOS DE ALTA EN LA SEGURIDAD SOCIAL POR COMUNIDADES AUTÓNOMAS. AÑOS 2007 Y 2013 (MEDIA DE DICIEMBRE)

\begin{tabular}{|c|c|c|c|c|c|c|c|}
\hline & \multicolumn{3}{|c|}{ Diciembre 2007} & \multicolumn{3}{|c|}{ Diciembre 2013} & \multirow{2}{*}{$\begin{array}{l}\text { Diferencia } \\
\text { porcentual } \\
2013 / 2007\end{array}$} \\
\hline & Total & Autónomo & $\%$ & Total & Autónomo & $\%$ & \\
\hline España & 63.399 & 20.501 & 32,3 & 89.786 & 42.559 & 47,4 & 15,1 \\
\hline Asturias & 512 & 244 & 47,6 & 703 & 458 & 64,6 & 17,0 \\
\hline Navarra (2008) & 385 & 195 & 50,6 & 673 & 406 & 60,3 & 9,7 \\
\hline Murcia & 1.381 & 491 & 35,5 & 1.973 & 1.185 & 60,1 & 24,6 \\
\hline La Rioja & 185 & 82 & 44,3 & 301 & 179 & 59,5 & 15,2 \\
\hline Aragon & 2.035 & 807 & 39,6 & 2.837 & 1.627 & 57,3 & 17,7 \\
\hline Galicia & 911 & 455 & 49,9 & 1.465 & 818 & 55,8 & 5,9 \\
\hline Castilla-Leon & 1.395 & 512 & 36,7 & 1.677 & 916 & 54,6 & 17,9 \\
\hline Castilla-La Mancha & 1.642 & 431 & 26,2 & 2.508 & 1.296 & 51,7 & 25,5 \\
\hline Ceuta & 60 & 22 & 36,7 & 89 & 46 & 51,7 & 15,0 \\
\hline Extremadura & 402 & 172 & 42,8 & 822 & 421 & 51,2 & 8,4 \\
\hline Andalucía & 6.360 & 2.244 & 35,3 & 9.981 & 5.063 & 50,7 & 15,4 \\
\hline Cantabria & 290 & 115 & 39,6 & 571 & 283 & 49,6 & 10,0 \\
\hline C. Valenciana & 6.467 & 2.677 & 41,4 & 9.479 & 4.706 & 49,6 & 8,2 \\
\hline País Vasco & 1.674 & 574 & 34,3 & 2.755 & 1.312 & 47,6 & 13,3 \\
\hline Madrid & 15.698 & 5.695 & 36,3 & 23.319 & 10.801 & 46,3 & 10,0 \\
\hline Cataluña & 18.600 & 4.269 & 22,9 & 23.920 & 10.575 & 44,2 & 21,3 \\
\hline Baleares & 1.642 & 477 & 29,0 & 1.874 & 772 & 41,2 & 12,2 \\
\hline Canarias & 3.794 & 1.064 & 28,0 & 4.787 & 1.681 & 35,1 & 7,1 \\
\hline Melilla & 33 & 13 & 39,4 & 55 & 14 & 25,4 & $-14,0$ \\
\hline
\end{tabular}

Fuente: Elaboración propia a partir de boletines mensuales de Afiliación de Extranjeros a la Seguridad Social. Secretaria de Estado de la Seguridad Social.

Donde han llegado más tarde los trabajadores y empresarios chinos es donde proporcionalmente más autónomos hay —Asturias, Navarra, Murcia, La Rioja, Aragón, Galicia-, porque se va allí directamente a abrir un negocio después de haber tenido una trayectoria previa de asalariado dentro del nicho étnico - formándose en la práctica en el sector y ahorrando capital para invertir en la 
apertura de un negocio propio- o en el mercado laboral general (Moraga Reyes, 2010; Rubio Ardanaz y Fang, 2014). Otro indicador que ayuda a entender esta dinámica es que los chinos son el grupo extranjero con una mayor tasa de movilidad residencial interprovincial, distanciándose mucho del resto en este comportamiento.

Por su parte, Madrid y Cataluña, las Comunidades Autónomas que concentran a más trabajadores y residentes chinos tradicionalmente, durante este periodo de cinco años, han tenido un comportamiento diferencial. En conjunto disminuye su peso sobre el total del trabajo chino en España, pasando de reunir del 54\% del total de los trabajadores al 52,6\%. Por su parte, mientras que Madrid en el año 2007 tenía 13,4 puntos porcentuales más de trabajadores autónomos chinos que Cataluña, en 2013 la diferencia se ha reducido a 2,1. Cataluña, Baleares, Canarias y Castilla-La Mancha en 2007 eran las Comunidades Autónomas que contaban con una proporción más baja de trabajadores autónomos chinos porque en ellas su situación de asalariados era mayor, bien en el nicho étnico o bien en el mercado laboral general. Castilla-La Mancha es la única de las anteriores que cinco años después prácticamente duplica su proporción de autónomos y se sitúa por encima de la media del 47,4\% (del $26,2 \%$ del total al $51,7 \%$ ), debido a las oportunidades para la apertura de nuevos negocios en la región, aunque también es significativo el avance de Murcia (24,6 puntos porcentuales más) y de Cataluña (21,3 puntos porcentuales).

\section{TRAYECTORIA EMPRESARIAL CHINA DURANTE LA CRISIS ECONÓMICA EN ESPAÑA}

En la exposición diacrónica anterior se ha analizado el proceso y la dinámica de la inserción económica de los chinos en España. Las características específicas de esta dinámica se han mantenido, consolidado y promovido durante el periodo de crisis, determinando un comportamiento laboral y empresarial caracterizado por 1) la continuidad y profundización de sus estrategias y expectativas; 2) la búsqueda, inserción y explotación de nuevos nichos económicos; 3) la intensificación del transnacionalismo en sus actividades empresariales (Beltrán y Sáiz, 2009). A continuación analizaremos con más detalle cada una de estas tendencias. 


\subsection{Continuidad y profundización de estrategias y expectativas previas}

El objetivo del proyecto migratorio chino procedente del distrito rural de Qingtian (provincia de Zhejiang) consiste en controlar y ser propietario de negocios, modelo de inserción económica seguido por la mayor parte de chinos procedentes de otros lugares. La empresa familiar es el medio para lograrlo, y el éxito social, el prestigio se obtiene al entrar a formar parte de la elite económica empresarial china. Para conseguir este objetivo se movilizan todo tipo de recursos y redes que facilitan tanto el proceso migratorio como el trabajo, la formación, la información, el capital, etc. Un conjunto de prácticas y saberes que conforman el locus de la cultura migratoria de esta zona.

Si no se dispone del capital inicial necesario para la inversión en la apertura de un negocio lo primero es su acumulación mediante el ahorro y/o el acceso a créditos, y/o la puesta en común de capital por parte de varios socios. Las tres posibilidades anteriores pueden o no coincidir, es decir una no excluye a las demás. En cualquier caso lo habitual es comenzar trabajando como asalariado dentro del nicho étnico o en el mercado laboral general. Durante esta fase el trabajo intensivo, largas jornadas laborales y una vida frugal son los elementos más característicos para una rápida acumulación de capital. Cuando se pone en común el ahorro producido gracias al esfuerzo de varios miembros de una misma familia durante varios años de trabajo asalariado, se puede llegar a disponer del capital necesario para invertir en un negocio que liberará a todos o a una parte de los miembros de la familia de su anterior situación de contratados para convertirse en la mano de obra de la nueva empresa familiar, pasando de una situación de dependencia laboral —régimen general o asalariado, o empleado sin contrato laboral en la economía sumergida - a otra de independencia — trabajador autónomo, empresario.

La crisis económica, en parte relacionada con la especulación inmobiliaria, ha tenido como consecuencia un abaratamiento del precio de la vivienda y de los locales comerciales, tanto de venta como de alquiler, así como del coste para realizar traspasos de negocios que están en funcionamiento. El abaratamiento de los costes ha repercutido en un mayor acceso al establecimiento de empresas familiares, es decir, más trabajadores chinos han pasado en menos tiempo a disponer del capital necesario para crear sus empresas y 
por eso su volumen de empresarios no ha parado de crecer, pasando del 32\% al 47\% de trabajadores autónomos durante estos seis años (2007-2013), al igual que se ha incrementado su residencia de un modo continuado. El volumen total de empresas, de comercios y de locales alquilados y vendidos en España muestra una tendencia a la baja durante la crisis. Sin embargo, los empresarios chinos con sus actividades e iniciativas económicas de apertura y traspasos de negocios han ayudado a que esa pérdida sea menor.

\subsection{Búsqueda, inserción y explotación de nuevos nichos económicos}

La inserción en la propiedad y gestión de bares, cafeterías y restaurantes autóctonos es una novedad que comienza en 2005 y para 2007 ya es una realidad que se va extendiendo desde las principales ciudades - Madrid y Barcelona- al resto de capitales de provincia y a otras ciudades grandes y medianas de toda la geografía nacional (Beltrán y Sáiz, 2013). Este es un sector que todavía no se ha saturado, y constituye un ejemplo de recambio étnico en una actividad con ausencia de recambio generacional. No obstante, se constata que los nuevos jóvenes empresarios chinos comparten con los autóctonos relevados las expectativas para con su descendencia: no desean que sus hijos e hijas continúen con este tipo de ocupación y tratan de invertir en su educación para que accedan en el futuro a otras alternativas laborales alejadas del trabajo intensivo característico de los bares.

Otros sectores de servicios donde se han insertado y se encuentran en pleno desarrollo y expansión son las peluquerías, que desde zonas muy concretas de concentración residencial china en las grandes ciudades, pues su clientela original era fundamentalmente coétnica, se han ido extendiendo al resto de la ciudad para una clientela general, así como se van estableciendo en ciudades medianas y pequeñas. Zapaterías y tiendas de ropa se han expandido también, aunque ya han encontrado el punto de saturación de su oferta.

En la búsqueda de nuevos nichos en la actualidad sobresalen las actividades emprendidas por los miembros más jóvenes del colectivo, a menudo componentes de la denominada segunda generación o generación uno y medio. Cada vez son más los que acceden a la Universidad y se gradúan en estudios superiores constituyendo 
unos recursos humanos bien formados y con conocimientos para emprender otro tipo de actividades empresariales de mayor valor añadido y menor trabajo intensivo. Mientras que algunos buscan un trabajo asalariado de "cuello blanco» bien pagado en puestos de la administración, grandes empresas, sector financiero, etc., otros ponen en juego su formación para diversificar las inversiones de la empresa familiar y se aventuran en otros ámbitos como la importación/exportación, la fabricación local de ropa de marca propias, asesorías para invertir en China, agencias de viaje y turismo, desarrollo de nuevas tecnologías de la información y comunicación, etc. De hecho, ellos están contribuyendo a configurar el actual mapa del espacio económico transnacional, al que se suman también el colectivo en aumento de los estudiantes de master y doctorado que proceden directamente de China y al graduarse trabajan y/o abren empresas de mayor valor añadido. Otro segmento emergente son los trabajadores de elevada cualificación enviados por empresas multinacionales chinas que comienzan a establecerse en España.

\subsection{Intensificación del transnacionalismo en la actividad empresarial}

Una alternativa empresarial, que se suma a las iniciativas ya emprendidas en España mediante el desarrollo de diferentes nichos étnicos, y que no excluye ni acaba con las ya existentes, es la inversión en actividades tanto productivas como de servicios e incluso de inversión especulativa en China. Algunos jóvenes chinos vuelven al país de los padres después de una experiencia más o menos larga de trabajo, y/o de gestión de una empresa, y/o con un éxito mayor o menor, en España. Otros incluso van a China después de graduarse en la universidad española para comenzar su actividad laboral directamente allí.

Si durante las distintas fases del ciclo de una empresa familiar apertura, consolidación, expansión- se ha constatado la importancia de los vínculos y actividades transnacionales (Sáiz López, 2013; Beltrán, 2010), así como la estrecha relación con el ciclo de vida familiar (Sáiz López, 2012), la vuelta a China se puede entender como una estrategia de diversificación de inversiones para evitar riesgos, así como una apuesta por nuevas oportunidades de negocios con expectativas de beneficios. El tipo de empresas en las que se invierte 
a veces son también de servicios — bares, cafeterías, restaurantesy pueden estar asociadas a la oferta de productos extranjeros, como por ejemplo la importación y venta de vino, aceite, jamón y otros productos españoles. También se invierte en la construcción y en el mercado inmobiliario chino, en agencias de viaje y turismo, en fabricación de ropa, en una amplia gama de empresas de importación/ exportación, etc. (Masdeu Torruella, 2014).

En ocasiones una parte de la familia continúa con sus negocios en España y otra pone en marcha nuevos negocios en China, y el trasvase de capital, información y mano de obra se moviliza en múltiples direcciones siguiendo los vínculos y espacios transnacionales. Una adaptación a la época de crisis es la diversificación de inversiones e iniciativas a escala internacional, sentando bases en varios países simultáneamente. De hecho esta ha sido siempre una característica de la élite económica china en el contexto migratorio: los más ricos eran los que tenían mayor diversificación de inversiones, de empresas y de presencia en más países diferentes en un momento dado. Ahora esta tendencia se encuentra al alcance de un mayor número de personas y familias, aunque el capital invertido y movilizado durante estos procesos sea menor.

\section{CONCLUSIONES}

Los chinos en España, en especial los qingtianeses que constituyen la mayoría, durante la crisis económica (2007-2013) han ido a contracorriente en el sentido de que el volumen de su población no ha dejado de aumentar, al igual que el número de sus empresas. La crisis ha coincidido con su inserción en un nuevo nicho económico -bares autóctonos-con la misma lógica de dispersión y expansión que ya pusieron en práctica previamente mediante otros nichos étnicos - restaurantes de comida china, bazares-. El abaratamiento de los costes para hacer frente al capital inicial y operativo de las empresas ha favorecido su constante apertura de nuevos negocios.

Además, la consolidación y el rápido desarrollo económico de China durante los últimos años han reforzado también las estrategias de los y las grandes empresarias que diversifican sus inversiones en varios países y sectores económicos sin abandonar su asentamiento español. Con y sin crisis, las personas chinas ya forman 
parte de la sociedad española. Su juventud y las expectativas y formación actual y futura de los mismos, pronto dará lugar a nuevos desarrollos empresariales de mayor valor añadido, contribuyendo al crecimiento de la economía española y de su internacionalización.

\section{BIBLIOGRAFÍA}

Beltrán Antolín, J. (2000): «La empresa familiar. Trabajo, redes sociales y familia en el colectivo chino». OFRIM /Suplementos, 6, pp. 129-153.

Beltrán Antolín, J. (2010): «Comunidades asiáticas en España: Movilidad transnacional en un territorio de frontera». Revista CIDOB d'Afers Internacionals, 92, pp. 15-37.

Beltrán Antolín, J. y Sáiz López, A. (2013): «Del restaurante chino al bar autóctono. Evolución del empresariado de origen chino en España y su compleja relación con la etnicidad», en M. Barros Nock y H. Valenzuela García, eds., Retos y estrategias del empresariado étnico. Estudios de caso de empresarios latinos en los Estados Unidos y empresarios inmigrantes en España. México, CIESAS, pp. 85-108.

Beltrán Antolín, J. y Sáiz López, A., eds. (2009): Empresariado asiático en España. Barcelona, Fundación CIDOB.

Ceccagno, A. (2007): «The Chinese in Italy at a Crossroads: The Economic Crisis», en Mette Thunø (ed) Beyond Chinatown, Copenhagen, NIAS, pp. 115-136.

Colectivo IOE (2012): Impactos de la crisis sobre la población inmigrante. Organización Internacional para las Migraciones-España.

Esteban, F.O. (2011): «Inmigración extranjera y crisis económica en España». Encrucijadas. Revista Crítica de Ciencias Sociales, 1, pp. 51-69.

GiL-Alonso, F y Domingo A. (2008): «La complementariedad de la ocupación de españoles y extranjeros: análisis sectorial y diferencias territoriales». Sistema. Revista de Ciencias Sociales, 206, pp. 21-47.

Light, I. (2006): «Economías étnicas», en J. Beltrán, L. Oso y N. Ribas (eds.) Empresariado étnico en España. Madrid, Ministerio de Trabajo y Asuntos Sociales, pp. 41-68.

Masdeu Torruella, I. (2014): Mobilities and Embodied Transnational Practices: An Ethnography of Return(s) and Other Intersections between China and Spain. Tesis doctoral. Universidad Autónoma de Barcelona.

Medina, E., Herrarte, A., y VicénS, J. (2010): «Inmigración y desempleo en España: impacto de la crisis económica». Información Comercial Española, 854, pp. 37-48. 
Moraga Reyes, J. (2010): Chinos en Asturias. La reciprocidad en el imperio del cálculo. Gijón, Muséu del Pueblu d’Asturies.

Oliver Alonso, J. (2013): «La inmigración y la doble recesión del mercado de trabajo en España 2011-12», en E. Aja, J. Arango y J. Oliver Alonso (dirs.), Inmigración y crisis: entre la continuidad y el cambio. Anuario de Inmigración en España (edición 2012). Barcelona, Fundación CIDOB, Diputació de Barcelona y Fundación Ortega-Marañón, pp. 28-58.

Oso, L. y Villares, M. (2005): «Mujeres inmigrantes y empresariado étnico: dominicanas en Madrid, argentinas y venezolanas en Galicia». Revista Galega de Economía, 14 (2), pp. 261-278.

Rubio Ardanaz, E. y Fang X. (2014): «Migrantes procedentes de China en Euskadi» en G. Moreno Márquez (ed.), Inmigración e impacto de la crisis. Anuario de la Inmigración en el País Vasco 2013. Bilbao, Ikuspegui, Servicio Editorial de la Universidad del País Vasco, 269-282.

SÁiz López, A. (2012): «Transnationalism, Motherhood, and Entrepreneurship: Chinese Women in Spain», en M. T. Segal, V. Demos y E. Ngan-ling Chow (eds.) Social Production and Reproduction at the Interface of Public and Private Spheres, Londres, Emerald, pp. 39-59.

SÁiz López, A. (2013): "Gender, Development and Asian Migration in Spain: the Chinese Case» en L. Oso y N. Ribas-Mateos (eds.) The International Handbook on Gender, Migration and Transnationalism: Global and Development Perspectives, Cheltenham y Northampton, Edward Elgar, pp. 165-184.

Solé, C. y Parella, S. (2009): «De asalariados a autoempleados. Una aproximación a las causas de las iniciativas empresariales de los inmigrantes en España». Revista Internacional de Organizaciones, 2, pp. 31-50.

Valero-Matas, J.A., Coca, J.R., Valero-Oteo, I. (2014): «Análisis de la inmigración en España y la crisis económica». Papeles de Población, 20 (80), pp. 9-45.

Veira, A., StaneK, M. y CAchón, L. (2011): «Los determinantes de la concentración étnica en el mercado laboral español». Revista Internacional de Sociología, 69 (M1), pp. 219-242.

Vidal-Coso, E., Gil-Alonso, F. y Domingo, A. (2012): «La destrucción de empleo de migrantes y españoles (2007-2011): Factores demográficos, sectoriales y territoriales». Papers de Demografía, 409.

Zugasti Mutilva, N. (2014): «Incidencia de la crisis en el empleo extranjero. Evidencias a partir de las transiciones laborales de trabajadores indefinidos». Papers. Revista de Sociologia, 99 (2), pp. 285-306. 